\title{
EVN OBSERVATIONS OF THE BINARY AGN IN THE MERGING GALAXY NGC 6240
}

\section{Y. Hagiwara*i}

National Astronomical Observatory of Japan

E-mail: yoshiaki.hagiwara@nao.ac.jp

\begin{abstract}
The results of VLBI observations of the nuclear region of the merging galaxy NGC 6240 obtained using the European VLBI Network (EVN) are presented. These new observations provided milliarcsecond (mas)-scale resolved images of the double radio nuclei at both $1.6 \mathrm{GHz}$ and $4.9 \mathrm{GHz}$ and revealed the spectral properties of these nuclei at this frequency range. The results of the observations coupled with earlier VLBI observations conducted at lower frequencies show hints of spectral turn over around at 2.4-4.9 GHz, which could be due to starburst or Active Galactic Nucleus (AGN) activities, and imply the presence of optically thick medium in both of the double nuclei.
\end{abstract}

The 9th European VLBI Network Symposium on the role of VLBI in the Golden Age for Radio Astronomy and EVN Users Meeting

September 23-26, 2008

Bologna, Italy

\footnotetext{
* Speaker.
} 


\section{Introduction}

NGC $6240(\mathrm{~d}=97 \mathrm{Mpc} ; \mathrm{Ho}=75 \mathrm{~km} / \mathrm{s} / \mathrm{Mpc})$ is a luminous infrared galaxy with puzzling morphological structure at all wavelengths due to forceful galactic collision of two galaxies with each nucleus. Earlier VLA observations and high-resolution MERLIN observations show that the two nuclei in the galaxy are separated by about 1.5 arcsecond [1, 2]. X-ray observations of the galaxy showed the presence of binary AGN toward the center of the galaxy [5]. The Very Long Baseline Array (VLBA) observations of these two nuclei both at 1.6 and $2.4 \mathrm{GHz}$ show high brightness temperatures of exceeding $10^{6} \mathrm{~K}$ for the nuclei [3]. The primary aim of new EVN experiments presented in this article was to obtain spectral indices of these nuclei at additional frequencies, 1.6, 2.3 and $8 \mathrm{GHz}$ observations having already been conducted with the VLBA [3].

\section{Observation}

The nuclear region of NGC 6240 was observed with the EVN at frequencies of $4.9 \mathrm{GHz}$ on 30 October 2003 and $1.6 \mathrm{GHz}$ on 10 November 2003. The participated telescopes were Effelsberg, Jodrell Bank (Lovell), Medicina, Noto, Westerbork (phased-array), Onsala, and Hartebeesthoek. The phase-referencing observations were conducted at the both frequencies using a nearby calibrator of J1651+0129. Correlation of the data took place at the JIVE using the EVN MkIV Data Processor.

\section{Results and discussions}

According to the preliminary data analysis, the positions of the detected radio sources by the EVN were identified with those of the double nuclei detected by VLBA in Gallimore and Beswick (2003) within a systematic error of approximately 1 mas. These sources were all detected with above $7 \sigma$ limits. These new EVN data are thus consistent with the earlier VLBA results and show the presence of high brightness components at each nucleus. The brightness temperature $(\mathrm{Tb})$ measured in the $4.9 \mathrm{GHz}$ observations are 3-6 x $10^{6} \mathrm{~K}$ for the both nuclei. The exceeding $\mathrm{Tb}$ values of $10^{6} \mathrm{~K}$ argue for an AGN origin of the both nuclei. The slightly extended structure of the northern nucleus $(\mathrm{N})$ obtained in the observations might suggest the core-jet structure that is typically seen towards AGN. The elongated structures of $\mathrm{N}$ at both the frequencies resemble jets in Seyfert galaxies on this scale. Radio power $(5 \mathrm{GHz})$ of $\mathrm{N}$ is about $10^{21} \mathrm{~W} / \mathrm{Hz}$, that corresponds to the range of Seyferts but too low for GPS or CSS [3]. The preliminary analysis of flux densities of the double nuclei reveals that the frequency turnover of the falls between $1.7 \mathrm{GHz}$ and $4.9 \mathrm{GHz}$.

The inverted spectra of the northern nuclei are due to free-free absorption or synchrotron selfabsorption (SSA) or a mixture of both. VLA/MERLIN observations at lower angular resolutions do not show frequency turnover, which means that absorbing media forefront the nuclei are very compact $(<10 \mathrm{pc})$, if the spectrum bending is due to free-free absorption. The northern nucleus could be therefore a highly obscured AGN but we cannot rule out radio supernovae for the northern nucleus. The southern nucleus (S) looks more compact and has a slightly larger brightness temperature. The southern nucleus is considered to be the most dominant AGN in the galaxy [5, 讨. The inverted spectrum between 1.7 and $2.4 \mathrm{GHz}$ shows steep spectrum index of 3.6. Given the compactness of the southern nucleus, SSA may affect the inverted spectrum, but free-free absorption 

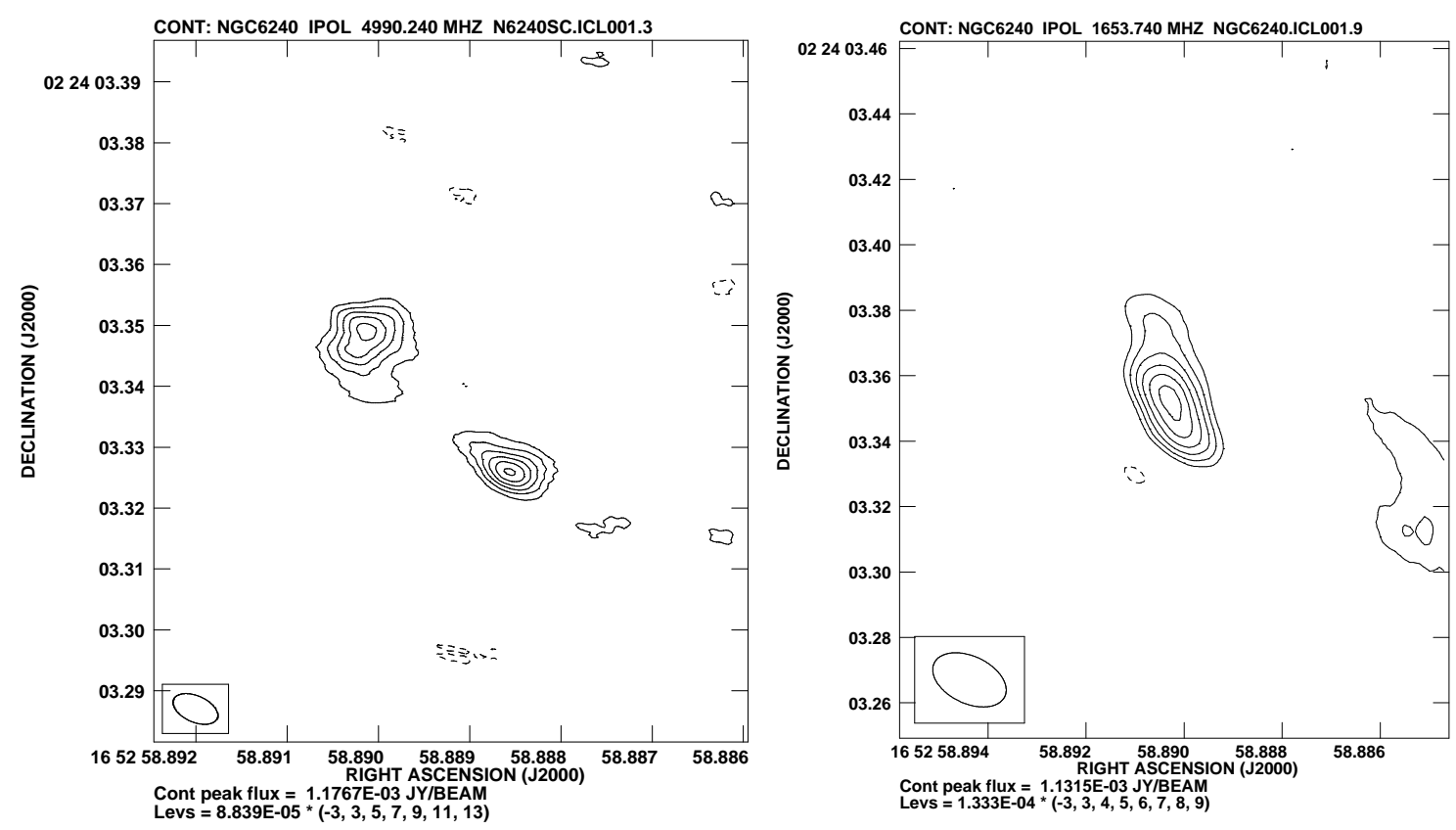

Figure 1: le ft: EVN 4.9 GHz image of the southern nucleus of NGC 6240 and an unresolved new component (Sn) associated with the nucleus, right: EVN 1.6 GHz image of the southern nucleus

cannot be ruled out [3].

On the other hand, a new compact source ( $\mathrm{Sn})$ at $4.9 \mathrm{GHz}$ has been identified in the southwest of the southern nucleus in Fig.1. Sn emerged about 30 mas (27 pc) offset from the southern nucleus in Fig.1 (right in the middle). The radio power of $3 \times 10^{21} \mathrm{~W} / \mathrm{Hz}$ of this source is similar to the brightest RSN in Arp 220 or in NGC 7469 [3]. In Fig.1, the $1.6 \mathrm{GHz}$ nucleus looks a single component with a slightly elongated structure in southwest but there seems no indication of a sub-component in the nuclear continuum structure, and $1.6 \mathrm{GHz}$ continuum emission is not seen at the southern part of $\mathrm{Sn}$ at $4.9 \mathrm{GHz}$. Accordingly, $\mathrm{Sn}$ is not clearly detected in the $1.6 \mathrm{GHz}$ image observed just 10 days after the $4.9 \mathrm{GHz}$ observations. The straightforward interpretation is that $\mathrm{Sn}$ is a RSN and will be brighter at lower frequencies following fading light curve.

\section{References}

[1] R. Beswick et al., MNRAS 325, 151 (2001)

[2] E. J. M. Colbert et al., ApJ 436, 89 (1994)

[3] J. Gallimore and R. Beswick, AJ 127, 239 (2003)

[4] Y. Hagiwara et al., A\&A 400, 457 (2003)

[5] S. Komossa et al., ApJ 582, L15 (2003) 